Hautarzt 2011 · 62:208-208

DOI 10.1007/s00105-011-2154-5

(C) Springer-Verlag 2011

\section{Bildung von reaktiven Sauerstoffspezies in vitro durch Malassezia-Hefen}

\section{Zusammenfassung des prämierten Beitrags}

\section{Zum Beitrag}

S. Später, U.-C. Hipler, U.-F. Haustein, P. Nenoff (2009) Bildung von reaktiven Sauerstoffspezies in vitro durch MalasseziaHefen. Hautarzt 60:122-127

\section{Hintergrund und Fragestellung}

Malassezia-Spezies sind Sprosspilze, die zur physiologischen Flora der Haut gehören und unter bestimmten Umständen Hauterkrankungen verursachen können. Eine Assoziation der Pilze ist u.a. mit der Pityriasis versicolor und dem seborrhoischen Ekzem nachgewiesen worden. Mittlerweile sind mindestens zwölf verschiedene Malassezia (M.)-Spezies bekannt, von denen eine Art - M. furfur - reaktive Sauerstoffspezies (reactive oxygen species, ROS) bildet. ROS sind freie Radikale, die eine toxische Wirkung auf Gewebe ausüben können. Die vorgestellte Studie untersuchte, ob neben M. furfur weitere Malassezia-Spezies die Fähigkeit zur Bildung von ROS haben. Zusätzlich wurde untersucht, inwieweit das Antimykotikum Terbinafin die ROS-Bildung beeinflusst.

\section{Versuchsaufbau}

Es wurden 7 Malassezia-Stämme aus Patientenisolaten und 4 Referenzstämme ausgewählt. Die untersuchten Isolate repräsentierten insgesamt 6 verschiedene Malassezia-Spezies: M. furfur, M. pachydermatis, M. sympodialis, M. obtusa, M. slooffiae und M. globosa. Die Pilze wurden in vitro auf Dixon-Agar kultiviert. Im Laufe des Zellwachstums wurde die gebildete ROSMenge durch Lucigenin-abhängige Che- milumineszenz bestimmt. Blastosporen der Stämme M. furfur und M. pachydermatis wurden zusätzlich für eine halbe Stunde mit verschiedenen Konzentrationen Terbinafin inkubiert, und es wurden die ROSMenge sowie das Zellwachstum bestimmt.

\section{Ergebnisse}

Für jede der untersuchten MalasseziaSpezies konnte die Bildung reaktiver Sauerstoffspezies nachgewiesen werden. Die gebildeten Sauerstoffradikale standen in linearer, proportionaler Abhängigkeit zur Sprosszelldichte. Die halbstündige Inkubation der $M$. furfur-Sporen mit $1 \mu \mathrm{g}$, $10 \mu \mathrm{g}$ oder $100 \mu \mathrm{g}$ Terbinafin hatte eine im Kontrollvergleich mit den unbehandelten Kulturen um 47 \%, 28 \% bzw. 23 \% verminderte ROS-Bildung zur Folge. Bei $M$. pachydermatis konnte ein ähnliches Ergebnis nachgewiesen werden. Eine Inkubation der Sprosspilze mit den genannten Terbinafin-Konzentrationen bewirkte eine Verminderung der ROS-Entwicklung um $24 \%$, 57,9 \% bzw. 35,9\%.

\section{Schlussfolgerungen}

Erstmals ist für sechs Malassezia-Spezies nachgewiesen worden, dass sie in der Lage sind, reaktive Sauerstoffspezies zu bilden. Die ROS-Bildung der Malassezia-Hefen könnte nach Meinung der Autoren ein weiterer Pathogenitätsfaktor sein und etwa beim seborrhoischen Ekzem und bei der Pityriasis versicolor rubra zu Entzündungsreaktionen beitragen. Die Intensität der ROS-Bildung hing in der vorgestellten Studie von der Zellzahl der Sprosspilze ab. Beim seborrhoischen Ekzem wurde im erkrankten Hautareal eine signifikant erhöhte Zellzahl von M. furfur und M. globo$s a$ nachgewiesen. Beim atopischen Ekzem scheint dagegen nicht die Zellzahl, sondern das Auftreten verschiedener MalasseziaArten nebeneinander eine Rolle zu spielen.

Das Antimykotikum Terbinafin reduziert die ROS-Bildung von $M$. furfur und M. pachydermatis in Abhängigkeit von der verwendeten Terbinafin-Konzentration. Möglicher Ansatzpunkt der antimykotischen Therapie mit Terbinafin könnte neben der bekannten Hemmung der Squalenepoxidase auch die Reduktion freier Sauerstoffradikale in mit Malassezia besiedelten erkrankten Hautarealen sein.

Bei niedrigen Konzentrationen des Antimykotikums (M. furfur nach Inkubation mit $1 \mu \mathrm{g} / \mathrm{ml}$ Terbinafin, $M$. pachydermatis nach Inkubation mit $10 \mu \mathrm{g} / \mathrm{ml}$ Terbinafin)wurde eine signifikante Reduktion der ROS erreicht, sodass weitere Versuche mit Terbinafin-Konzentrationen zwischen $1 \mu \mathrm{g} / \mathrm{ml}$ und $10 \mu \mathrm{g} / \mathrm{ml}$ durchgeführt werden sollten.

Inwieweit dem Ergebnis der ROS-Reduktion bei $M$. pachydermatis für den Menschen praktische Relevanz zukommt, bleibt fraglich, weil M. pachydermatis nur in Ausnahmefällen isoliert wird. Eventuell könnte der Effekt der ROS-Reduktion aber in der Veterinärmedizin, z.B. bei der Behandlung der Otitis externa des Hundes, eine Anwendung finden.

\section{Mehr Information ?}

Sie können den in Hautarzt Heft 2/09 veröffentlichten Beitrag in voller Länge im Internet unter www.DerHautarzt.de (Inhalt/ Archiv) abrufen. 